\title{
Heavy Weyl Fermion State in $\mathrm{CeRu}_{4} \mathrm{Sn}_{6}$
}

\author{
Yuanfeng Xu, ${ }^{1}$ Changming Yue, ${ }^{1}$ Hongming Weng, ${ }^{1,2}$ and Xi Dai ${ }^{1,2, *}$ \\ ${ }^{1}$ Beijing National Laboratory for Condensed Matter Physics, and Institute of Physics, \\ Chinese Academy of Sciences, Beijing 100190, China \\ ${ }^{2}$ Collaborative Innovation Center of Quantum Matter, Beijing 100084, China \\ (Received 20 August 2016; revised manuscript received 16 December 2016; published 7 March 2017)
}

\begin{abstract}
A new type of topological state in strongly correlated condensed matter systems, the heavy Weyl fermion state, has been found in a heavy fermion material, $\mathrm{CeRu}_{4} \mathrm{Sn}_{6}$, which has no inversion symmetry. Two different types of Weyl points, types I and II, can be found in the quasiparticle band structure obtained by the LDA + Gutzwiller calculations, which can treat the strong correlation effects among the $f$ electrons from cerium atoms. The surface calculations indicate that the topologically protected Fermi arc states exist on the (010) but not on the (001) surface.
\end{abstract}

DOI: 10.1103/PhysRevX.7.011027

Subject Areas: Condensed Matter Physics, Strongly Correlated Materials, Topological Insulators

\section{INTRODUCTION}

Recently, different types of topological semimetals [1] have been proposed and observed in condensed matter systems, i.e., the Weyl semimetal (WSM) phase in transition metal compounds with magnetic order [2,3], the WSM phase in noncentral symmetric crystals [4-9], the Dirac semimetal (DSM) phase in intermetallic compounds [10-15], and the nodal line semimetal phase in antiperovskite compounds $[16,17]$. In all the abovementioned material systems, the electron-electron correlation effect is weak, and the band structure, as well as the existence of Weyl nodes, can be obtained quite accurately by density functional theory. On the other hand, the topological nontrivial electronic structure can be found in strongly correlated material systems as well; for instance, the topological Kondo insulator phase in $\mathrm{SmB}_{6}[18-20]$ can be viewed as the strongly correlated $Z_{2}$ topological insulator, which has attracted much interest in recent years [21-25]. In $\mathrm{SmB}_{6}$, the correlation effects generated by the strong Coulomb repulsive interaction among $f$ electrons suppress the bandwidth dramatically but leave the topological features of the electronic structure unchanged [20,24,26,27].

In the present work, we propose that $\mathrm{CeRu}_{4} \mathrm{Sn}_{6}$ [28-33], a typical heavy fermion material, contains Weyl points in its quasiparticle band structure near the Fermi level and thus belongs to a new class of strongly

*daix@iphy.ac.cn

Published by the American Physical Society under the terms of the Creative Commons Attribution 4.0 International license. Further distribution of this work must maintain attribution to the author(s) and the published article's title, journal citation, and DOI. correlated topological-phase, heavy Weyl fermion states. Comparing to other WSMs found in noninteracting systems, the WSM phase in heavy fermion systems has more fruitful physical properties because of the following reasons. First, unlike the noninteracting systems, the heavy quasiparticle bands are fully developed only at low temperatures. Therefore, how the physics related to the topological electronic structure evolves as the decrement of temperature will become a crucial problem for the heavy Weyl fermion phase, which may lead to new unique phenomena in these systems. Second, in heavy fermion systems, the energy scale of the quasiparticle bands is orders smaller than the ordinary semiconductor or semimetal systems, which makes it more sensitive to various external fields, i.e., the pressure, magnetic field, and strain, providing large tunability for the distribution of the Weyl nodes.

The crystal structure of $\mathrm{CeRu}_{4} \mathrm{Sn}_{6}$ [28] has no inversion center, and the spin-orbit coupling (SOC) derived from the $4 f$ orbitals of cerium and $4 d$ orbitals of $\mathrm{Ru}$ splits the quasiparticle bands, which makes it possible to have Weyl points near the Fermi level. The electronic structure of $\mathrm{CeRu}_{4} \mathrm{Sn}_{6}$ has been studied both theoretically and experimentally by several groups already [30-35]. As introduced in Refs. [31,32], the band structure obtained by generalized gradient approximation (GGA) is a semimetal type, with a vanishing indirect but finite direct energy gap between the valence band mostly consisting of ruthenium $4 d$ orbitals and a conduction band formed mainly by the cerium $4 f$ orbitals. The LDA + DMFT calculation has been applied to this material by Held's group to capture the strong correlation effects [33]. Besides the reduction of the bandwidth for the $f$ bands, another significant consequence of the correlation effect is to enhance the crystal splitting within the $J=5 / 2$ subspace and push down the lowest $4 f$ 
bands with the $\left|J=5 / 2 ; J_{z}= \pm 1 / 2\right\rangle$ character, which leads to "inverted features" between $4 f$ and $4 d$ bands in some areas of the BZ mimicking the situation in $\mathrm{SmB}_{6}$. This picture has been partly supported by the $\mathrm{x}$-ray absorption and scattering data, as discussed in Ref. [32].

The previous studies imply that $\mathrm{CeRu}_{4} \mathrm{Sn}_{6}$ might be another topological heavy fermion material because of the band inversion features between bands with $4 f$ and $5 d$ character. While unlike the situation in $\mathrm{SmB}_{6}$, in $\mathrm{CeRu}_{4} \mathrm{Sn}_{6}$, because of the lack of inversion center, there is no symmetry or topological principle that can guarantee the opening of a full insulating gap after the band inversion. It may become a topological insulator if the gap is fully opened, or a WSM if the gap remains closed only at some isolated Weyl points (WP). Therefore, careful computational studies with reliable methods are needed to reveal the topological nature of its electronic structure. In the present paper, by using the LDA + Gutzwiller method [36], we have studied the renormalized quasiparticle band structure of $\mathrm{CeRu}_{4} \mathrm{Sn}_{6}$. Our results reveal that it contains Weyl points formed by heavy fermions and thus belongs to a new class of topological phases, the heavy WSM. Further calculations on different surfaces indicate that because of the existence of the three-dimensional bulk Fermi surfaces, protected surfaces states (SS) do not exist on the (001) surface, but they do exist on the (010) surface, where the projections of the bulk Fermi surfaces are well separated. Our LDA + Gutzwiller calculation then reveals a very long and beautiful Fermi arc pattern on the (010) surface, which can be detected by various experiments, including angleresolved photoemission spectroscopy (ARPES) and quantum oscillation.

\section{COMPUTATIONAL DETAILS}

$\mathrm{CeRu}_{4} \mathrm{Sn}_{6}$ is crystalized in a body-centered tetragonal lattice with a space group of $I \overline{4} 2 m$ (No. 121), which has no inversion center (Fig. 1). The experimental lattice constants $a=6.8810 \AA$ and $c=9.7520 \AA$ are adopted in our calculation [28]. The $\mathrm{Ce}$ and $\mathrm{Ru}$ atoms are located at Wyckoff positions $2 a(0.0,0.0,0.0)$ and $8 i(0.82938$, $0.82938,0.42107)$, respectively. The $\mathrm{Sn}$ atoms take two Wyckoff positions: $8 i(0.82134,0.82134,0.70476)$ and $4 c(0.0,0.5,0.0)$. In the present work, we first perform the electronic structure calculation by using the Vienna ab initio Simulation Package (VASP) [37] with the PBE-GGAtype exchange correlation potential. The plane-wave cutoff energy is $410 \mathrm{eV}$. Second, the maximally localized Wannier functions for $4 f$ and $5 d$ orbitals on Ce, $4 d$ orbitals on $\mathrm{Ru}$, and $5 p$ orbitals on $\mathrm{Sn}$ atoms have been constructed by using the Wannier90 package [38]. The SOC strength is obtained by fitting to the corresponding full-relativistic first-principles calculations, which are $0.096 \mathrm{eV}$ for $\mathrm{Ce} 4 f$, $0.160 \mathrm{eV}$ for $\mathrm{Ru} 4 d$, and $0.202 \mathrm{eV}$ for $\mathrm{Sn} 5 p$ orbitals. The on-site interactions among the partially occupied $f$ orbitals are crucial, and they can be properly treated by the LDA + Gutzwiller method [20,24,36]. We take the Coulomb interaction $U_{d}$ of $5.0 \mathrm{eV}$ and Hund's rule coupling $J_{h}$ of $0.818 \mathrm{eV}$ [39]. Similar to other post LDA methods, i.e., LDA + U and LDA + DMFT, the total Hamiltonian can be written as

$$
H_{\text {total }}=H_{\mathrm{GGA}}+H_{\mathrm{SOC}}+H_{\mathrm{int}}+H_{\mathrm{DC}}
$$

with $H_{\mathrm{GGA}}, H_{\mathrm{SOC}}$, and $H_{\text {int }}$ being the noninteracting Hamiltonian obtained by GGA, the atomic spin-orbital coupling, and the interacting Hamiltonians described above. The last term in Eq. (1) is the double-counting Hamiltonian, which needs to be included to remove the local interaction energy treated by GGA already in the mean-field manner. In the present study, the fully localized limit scheme is adopted for the double-counting energy [40].

In the LDA + Gutzwiller method, the Gutzwiller-type wave function $|G\rangle=\hat{P}|0\rangle$ has been proposed for the trial (a)

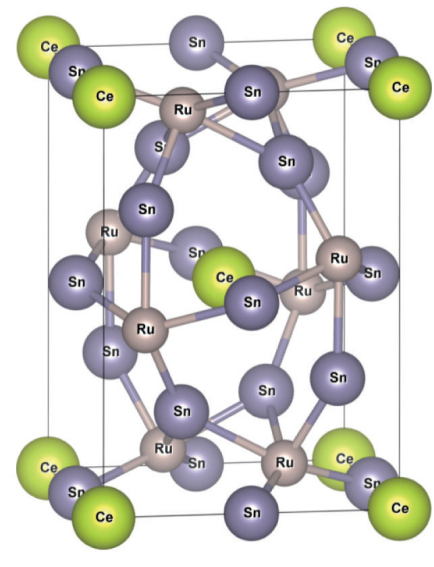

(b)

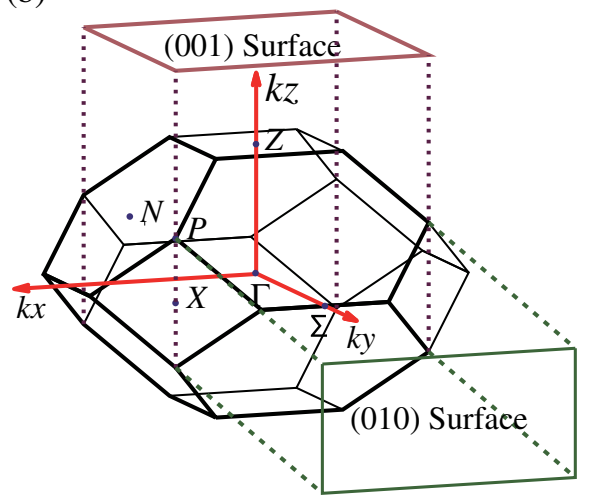

FIG. 1. Crystal structure and Brillouin zone (BZ). (a) The crystal symmetry of $\mathrm{CeRu}_{4} \mathrm{Sn}_{6}$. (b) The bulk BZ and the projected surface BZ for both (001) and (010) surfaces. 
wave function to minimize the ground-state energy, where $|0\rangle$ is the noninteracting wave function and $\hat{P}$ is the local projector applied to adjust the probability of the local atomic configuration. In addition, the Gutzwiller approximation is applied to evaluate the ground-state energy, and an effective Hamiltonian $H_{\mathrm{eff}} \approx \hat{P} H_{\mathrm{GGA}} \hat{P}$ describing the quasiparticle dispersion can be obtained, from which the possible Weyl points can be found. For a detailed description of the method, please refer to Refs. [36,41,42].

\section{RESULTS AND DISCUSSION}

Band structure of $\mathrm{CeRu}_{4} \mathrm{Sn}_{6}$. - The GGA band structure of $\mathrm{CeRu}_{4} \mathrm{Sn}_{6}$ is calculated and plotted in Fig. 2(a) with the SOC being fully considered, which is a semimetal type with very slight overlap between conduction and valence bands. The direct gap remains finite throughout the whole BZ at the GGA level. Because of the strong repulsive interaction among $f$ electrons, the quasiparticle bands mainly with $4 f$ character will be strongly renormalized and only
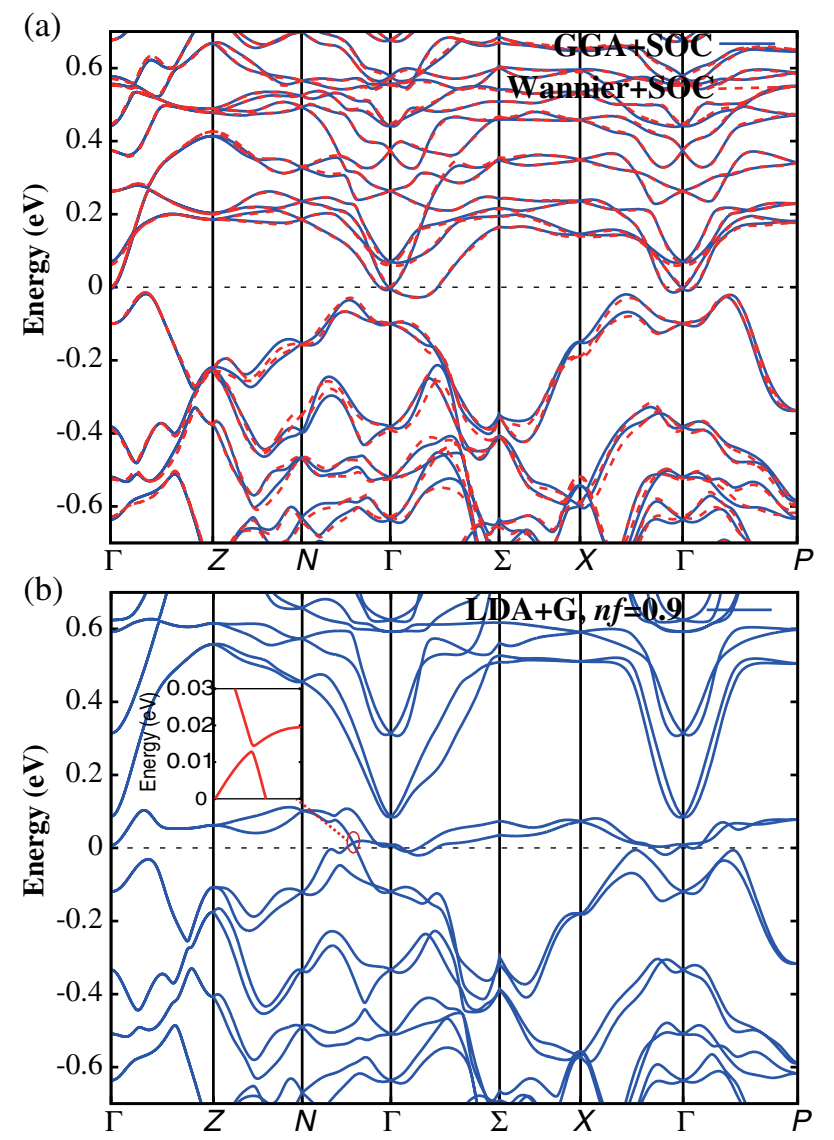

FIG. 2. (a) The band structure of $\mathrm{CeRu}_{4} \mathrm{Sn}_{6}$ calculated by GGA + SOC (solid line) and Wannier + SOC (dashed line). (b) The renormalized band structure of $\mathrm{CeRu}_{4} \mathrm{Sn}_{6}$ calculated by the LDA + Gutzwiller method. The Fermi energy is set to $0 \mathrm{eV}$. The gap of the bands in the inset is about $1.5 \mathrm{meV}$, which indicates that a zero gap Weyl point exists nearby. appear below some certain temperature scale (the Kondo temperature), below which the $f$ electrons start to participate in the coherent motion. In the present study, we apply the LDA + Gutzwiller method to quantitatively calculate the renormalized quasiparticle bands, which are plotted in Fig. 2(b). Compared to the GGA band structure, there are two major corrections caused by the strong correlation effects. First, the total bandwidth of the $4 f$ bands has been suppressed by approximately 50\%. Second, and more importantly for this particular material, the splitting among the $4 f$ orbitals has been greatly enhanced, leading to two direct consequences: The bands with $\mid J=5 / 2 ; J_{z}=$ $\pm 1 / 2\rangle$ character are pushed down to mix strongly with the $4 d$ bands from the $\mathrm{Ru}$ atoms; meanwhile, the bands with $|J=7 / 2\rangle$ character are pushed up to about $1.2 \mathrm{eV}$ above the Fermi level. Compared with the previous DMFT study [33], our renormalized band structure is in very good agreement with their results. The histograms of the atomic configurations in the ground state can be obtained by the LDA + Gutzwiller method and are plotted in Fig. 6(d), from which one can estimate the average occupation number of the cerium $4 f$ orbitals to be 0.9 . These results are quite consistent with the recent $\mathrm{x}$-ray absorption data suggesting the maximum occupation of the $4 f$ orbitals to be 0.95 .

Weyl nodes in $\mathrm{CeRu}_{4} \mathrm{Sn}_{6}$.-Because of the lack of an inversion center, all the quasiparticle bands are nondegenerate away from the eight time-reversal-invariant $k$ points. Point group symmetry analysis indicates that the mixing of the $\left|J=5 / 2 ; J_{z}= \pm 1 / 2\right\rangle$ and the $4 d$ states near the Fermi level will open a hybridization gap between them along any high symmetry lines, as we see in Fig. 2(b). At generic $k$ points, there is no symmetry that can protect the gapless nodes, but in three-dimensional space, accidental degeneracy can appear between the energy bands without spin degeneracy and lead to the appearance of WPs. WPs are monopoles with positive or negative chirality for the Berry curvature calculated from all the occupied bands, which satisfies Gauss's law. Therefore, the integral of the Berry curvature on any closed surface in the BZ will give us the total chirality of the WPs enclosed inside it, which can help us confirm the existence of the WP quickly.

The precise positions for the WPs have been obtained by LDA + Gutzwiller calculations on a much denser grid in $k$ space. As shown in Figs. 3(e) and 3(f), the WPs with positive and negative chirality can be identified as the "source" and "drain" of the Berry curvature. In total, there are eight pairs of WPs, as illustrated in Figs. 3(b)3(d) and Table I, which can be divided into two groups labeled as $W_{1}$ and $W_{2}$ so that the WPs in each group can be linked by crystal symmetry. The energy dispersion along the direction connecting $W_{1}$ and $W_{2}$ (as illustrated in Table I) has been plotted in Fig. 3(a), which clearly indicates that $W_{2}$ represents type-II WPs whereas $W_{1}$ is type I. The coexistence of the two different types of WPs 

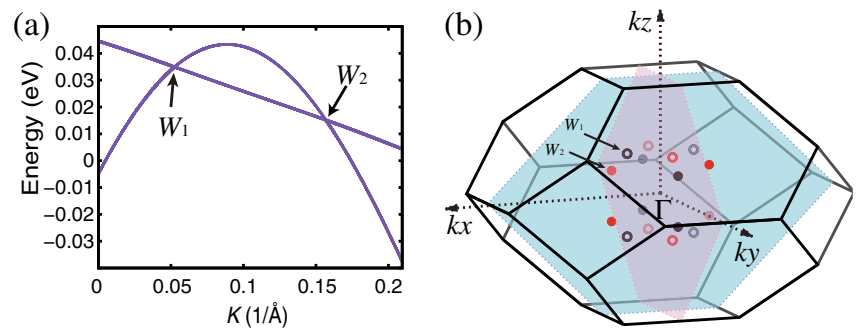

(c)

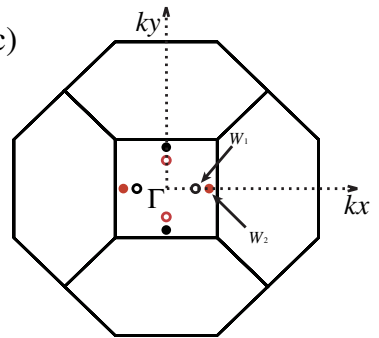

(d)

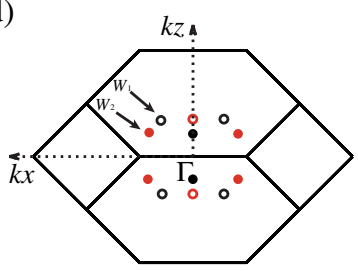

(e)
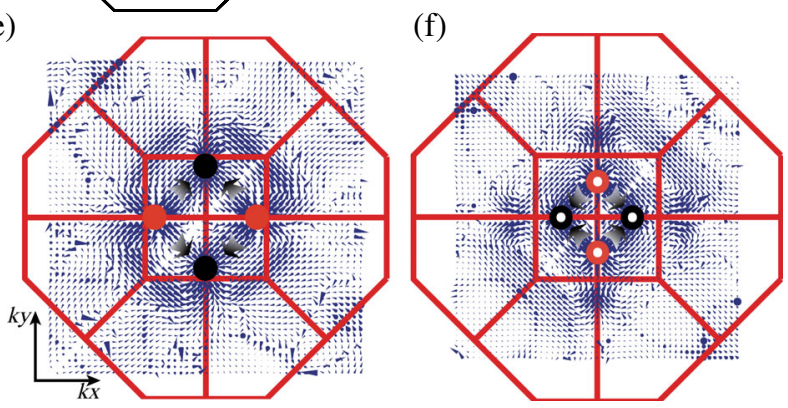

FIG. 3. (a) The energy dispersion along the direction connecting $W_{1}$ and $W_{2}$. (b) Three-dimensional view of the eight pairs of WPs in the BZ. (c) Top view from [001] and (d) side view from [010] directions for the WPs. The hollow and solid dots represent WPs in the $W_{1}$ and $W_{2}$ groups, respectively. (e) The distribution of Berry curvature for the $k_{z}=0.139 \AA^{-1}$ plane. (f) The distribution of Berry curvature for the $k_{z}=0.227 \AA^{-1}$ plane. The black and red dots denote the Weyl points with negative and positive chirality, respectively.

has been discussed recently in Ref. [43], and here we find the first realistic material that belongs to that category and thus to a new class of topological phases, the heavy WSMs. The splitting distance of a pair of WPs with different chirality in $\mathrm{CeRu}_{4} \mathrm{Sn}_{6}$ is about $0.1 \AA^{-1}$, which is about 4 times that in TaAs [4]. Thus, the WPs

TABLE I. The positions $(k x, k y, k z)$ in $k$ space of the two nonequivalent Weyl points in the BZ. The other seven pairs of Weyl points are related to the ones listed with reflections located at $(k x, k y,-k z)$ and $(-k x, k y, \pm k z)$ with the same energy and chirality as $(k x, k y, k z)$, and $(k y, \pm k x, \pm k z)$ with the same energy but opposite chirality relative to $(k x, k y, k z)$. Fermi energy is set to $0 \mathrm{eV}$.

\begin{tabular}{lccc}
\hline \hline Weyl point & $(k x, k y, k z)\left(\AA^{-1}\right)$ & Chirality & $E(\mathrm{meV})$ \\
\hline$W_{1}$ & $(0.132,0.0,0.227)$ & -1 & 35 \\
$W_{2}$ & $(0.193,0.0,0.139)$ & +1 & 15 \\
\hline \hline
\end{tabular}

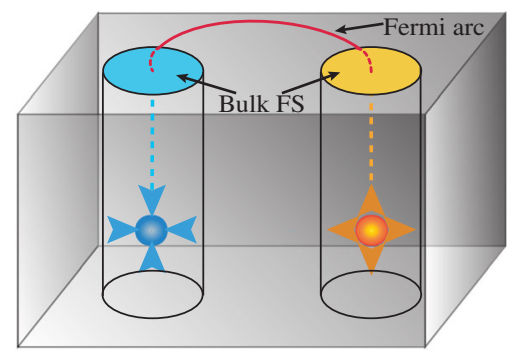

FIG. 4. A pair of Weyl points with opposite chirality projected onto the surface of the Brillouin zone along one direction and connected by the Fermi arc. If the Fermi surface of the bulk state and the Fermi arc are projected together, the Fermi arc will connect two bulk Fermi surfaces, each of which covers a Weyl point.

are very robust and will not be difficult for ARPES to detect.

Surface states of $\mathrm{CeRu}_{4} \mathrm{Sn}_{6}$. - In an ideal WSM, the Fermi level only cuts the WPs, and the Fermi surface (FS) of the bulk states are isolated points, which will generate protected Fermi arcs on any surface as long as the projection points of the WPs on that particular surface $\mathrm{BZ}$ are separated from each other [2,3]. In realistic WSM materials, there are finite-size bulk FS as well, which project to the surface BZ as ellipses enclosing different projection points of the WPs. Then, following the proof in Ref. [2], the topologically protected Fermi arc will still exist when these ellipses do not overlap each other, as schematically shown in Fig. 4. In $\mathrm{CeRu}_{4} \mathrm{Sn}_{6}$, the finitebulk FSs do exist, and they project onto the surface BZ of (001) and (010) surfaces, as shown in Fig. 5. Interestingly, although these projection areas overlap on the (001) surfaces, they are well separated on the (010) surfaces, which leads to the protected Fermi arcs existing on the (010) surface but not the (001) surface. We further calculated the surface states (SS) on both (001) and (010) surfaces with the software package Wannier_tools [44] using the renormalized effective tight-binding Hamiltonian obtained by LDA + Gutzwiller together with the Green's function method. As shown in Figs. 5(a) and 5(b), there are no clear SS on the (001) surface because of the overlap of the bulk FS. While for the surfaces along the (010) direction the situation is very different, there are very long and clear Fermi arcs connecting WPs with opposite chirality, as plotted in Figs. 5(c) and 5(d).

We emphasize that the existence of Fermi arcs on (010) and (100) surfaces is completely determined and protected by the topology of its bulk band structure. Therefore, they are robust against any change on these surfaces, i.e., the different surface termination and possible surface potential caused by charge accumulation on these surfaces, which can only change the connecting pattern of Fermi arcs but not annihilate them. In fact, we can define a topological invariance for any possible connection pattern of Fermi 

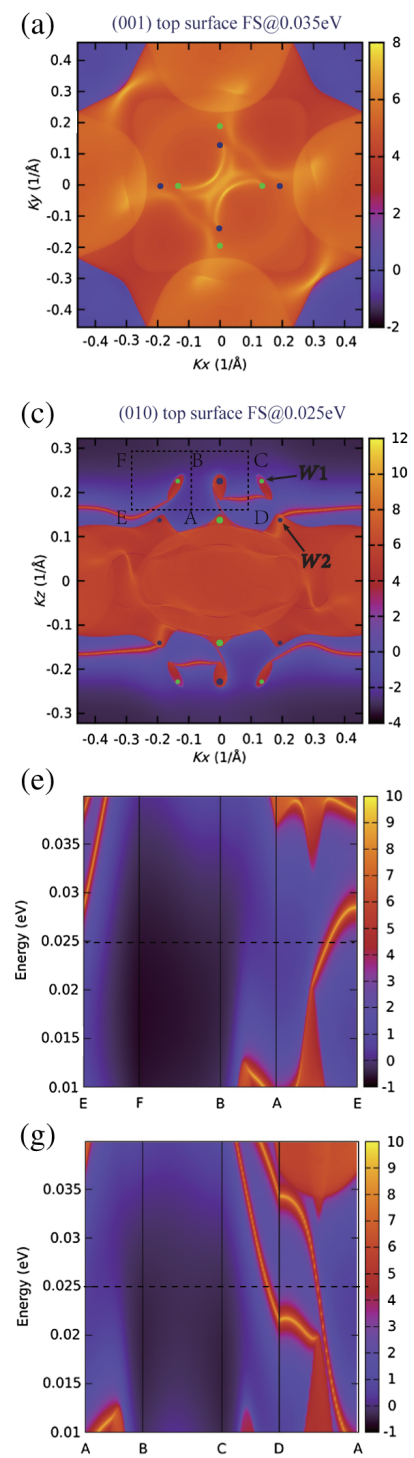
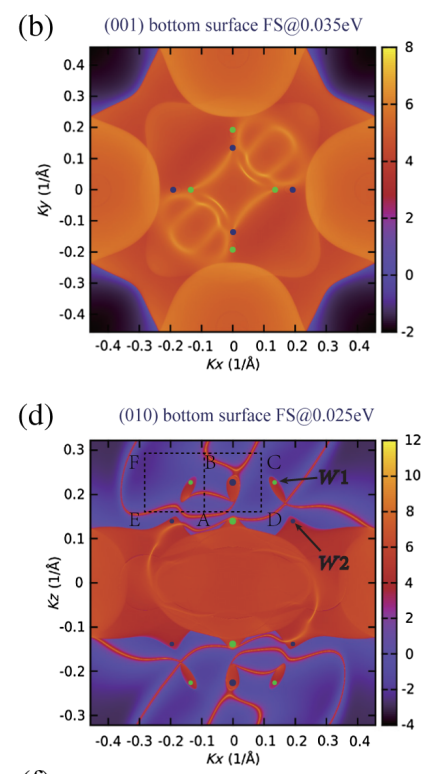

(f)

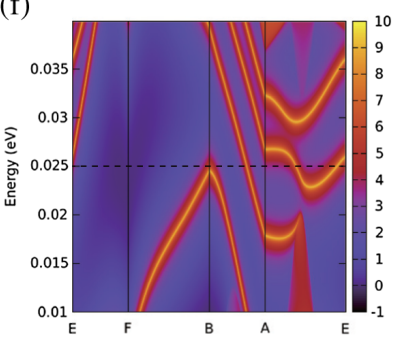

(h)

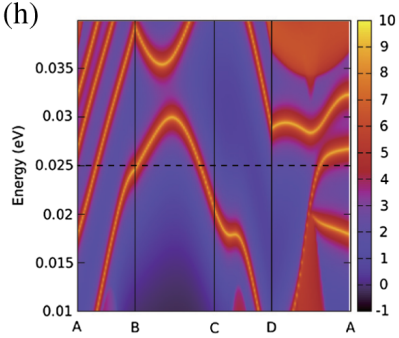

FIG. 5. Fermi surface on (a) top and (b) bottom (001) surfaces of the sample. All the dots represent two WPs with the same chirality projecting on top of each other. (c,d) Fermi surface on the top and bottom (010) surfaces, respectively. The small dots represent the single projected WP, and the large dots represent two WPs with the same chirality projecting on top of each other. Panels (e) and (g) are the surface states of the top surface along the closed paths of E-F-B-A-E and A-B-C-D-A, respectively. Panels (f) and (h) are the same as (e) and (g) but for the bottom surface.

arcs, which is the summation of the chirality of all the Fermi surfaces crossing points along any closed loops in the surface BZ. It is easy to prove that as long as the loop is away from the projection area of the bulk FS, this number must equal the total chirality of the WPs that are projected insides the loop and will not change under any modification of the surface condition. As an example, we have plotted the surface band dispersion on four different closed loops in Figs. 5(e)-5(h), which illustrates the above topological

invariance of Fermi arcs on both top and bottom surfaces. In addition, we have also calculated the surface states on another possible surface termination and with different surface potentials as described in detail in Appendixes B and $\mathrm{C}$, which clearly shows the robustness of the abovementioned surface topological invariance. Although the direct detection of WPs in the bulk band structure is difficult, it is relatively easy to detect those exotic SSs on (010) surfaces, which will lead to abnormal physical properties and can be detected by ARPES, transport, and quantum oscillation experiments.

Phase diagram and tunability of $\mathrm{CeRu}_{4} \mathrm{Sn}_{6}$. - The total number and location of the WPs are quite sensitive to the detail of the band structures. In the present study, although the interaction parameters as well as the doublecounting scheme adopted in the LDA + Gutzwiller calculations are all reasonable, leading to consistent results with the previous numerical and experimental studies, a small change of the band structure may still lead to a qualitative change of the WPs. Therefore, it is important to further check the robustness of the heavy Weyl fermion state against the small uncertainty from the numerical calculations, which is unavoidable at the current stage. Compared to the change of interaction parameters, the quasiparticle band structure is more sensitive to the change
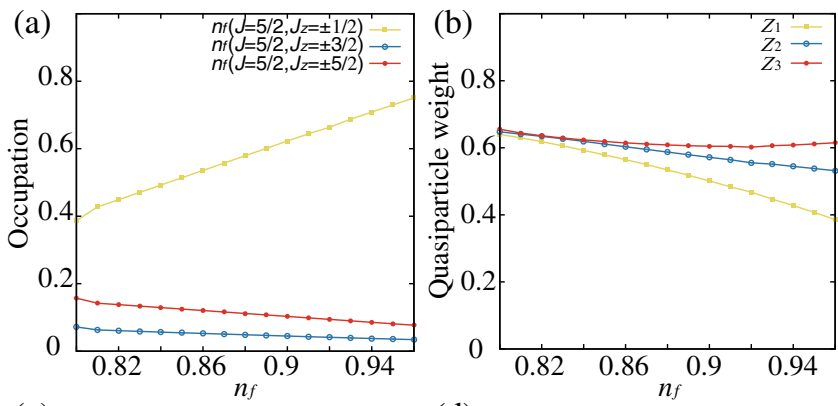

(c)

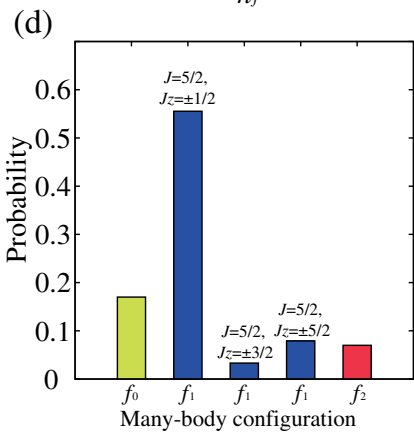

FIG. 6. (a) The occupation of $f$ orbitals with $J=5 / 2$, $J_{z}= \pm 1 / 2, \pm 3 / 2, \pm 5 / 2$ characters; (b) the quasiparticle weights for the $4 f$ orbitals with $J=5 / 2$; (c) phase diagram of $\mathrm{CeRu}_{4} \mathrm{Sn}_{6}$, with a change of the total $4 f$ orbital occupation number. The violet and green regions are semiconductor (SC) and semimetal (SM) phases. The blue and yellow regions are WSM phases with 8 and 12 pairs of Weyl points, respectively. (d) The histogram of the atomic configurations for the case of $n_{f}=0.9$. 
of the double-counting potential, which slightly modifies the physical valence of the cerium ions. Therefore, in the present paper, we calculate different quasiparticle band structures with the modification of the double-counting potential and plotted the phase diagram with the resulting occupation of the $4 f$ orbitals in Fig. 6(c). Our results indicate that the existence of the heavy Weyl fermion state in this material is very robust; it appears when $n_{f}>0.87$. At physical occupation $n_{f}=0.9$, the electronic structure of $\mathrm{CeRu}_{4} \mathrm{Sn}_{6}$ contains eight pairs of WPs, while for $n_{f}>0.92$, the number of WPs increases to twelve pairs, which leads to more complicated Fermi arc patterns on the surfaces.

The above phase diagram also indicates that compared to the WSM state found in weakly correlated materials, the properties of the heavy Weyl fermion state introduced here are much more sensitive to the external fields that can modify the effective valence of cerium-for instance, the pressure, strain, and chemical doping, which provide great tunability in this system and make it a promising material platform for follow-up studies on the relationship between correlation and topology.

\section{CONCLUSION}

In conclusion, based on the LDA + Gutzwiller calculation, we find that $\mathrm{CeRu}_{4} \mathrm{Sn}_{6}$ is the first Weyl semimetal in heavy fermion materials. The unique electronic structure of $\mathrm{CeRu}_{4} \mathrm{Sn}_{6}$ is greatly renormalized by the strong correlation effects among the $f$ electrons, leading to the appearance of WPs in the heavy quasiparticle bands. The surface calculations indicate that the Fermi arcs on the (010) surface are well separated from the projection of the bulk bands and thus can be detected by ARPES or quantum oscillation experiments.

\section{ACKNOWLEDGMENTS}

We acknowledge support from the National Key Research and Development Program of China (Grant No. 2016YFA0300600), the National 973 program of China (Grant No. 2013CB921700), the National Natural Science Foundation of China (Grants No. 11674369 and No. 11422428), and the "Strategic Priority Research Program (B)" of the Chinese Academy of Sciences (Grant No. XDB07020100) and Science Challenge Project. Some of the calculations were preformed on TianHe-1(A), the National Supercomputer Center in Tianjin, China.

\section{APPENDIX A: TWO NONEQUIVALENT CLEAVED CELLS OF CeRu $\mathrm{Sn}_{6}$}

There are two possible cleavage planes for $\mathrm{CeRu}_{4} \mathrm{Sn}_{6}$ single crystals along the (010) direction, which are

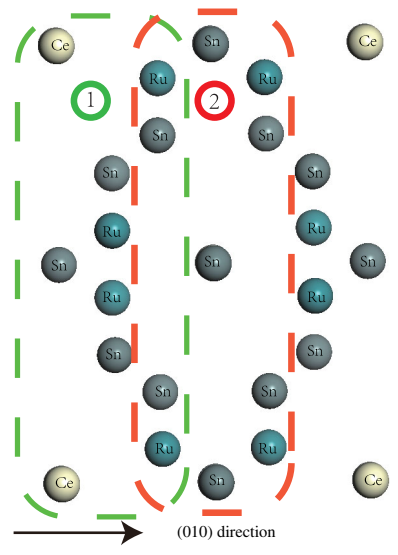

FIG. 7. Two different cleavage surface layers in the (010) direction enclosed by the green and red dashed lines.

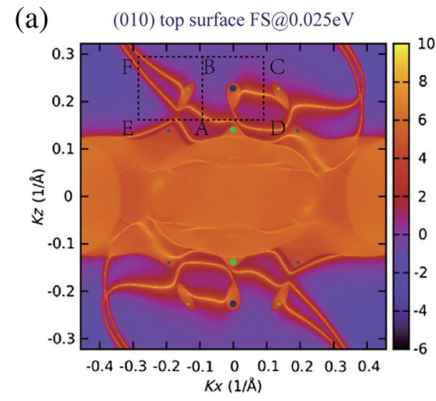

(b) (010) bottom surface FS@0.025eV
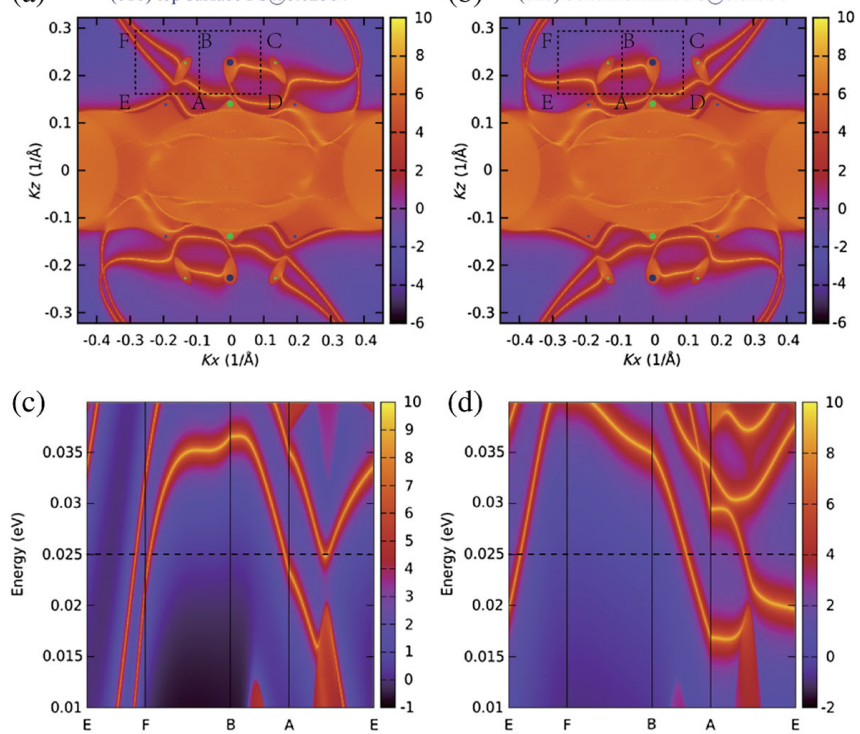

(e)
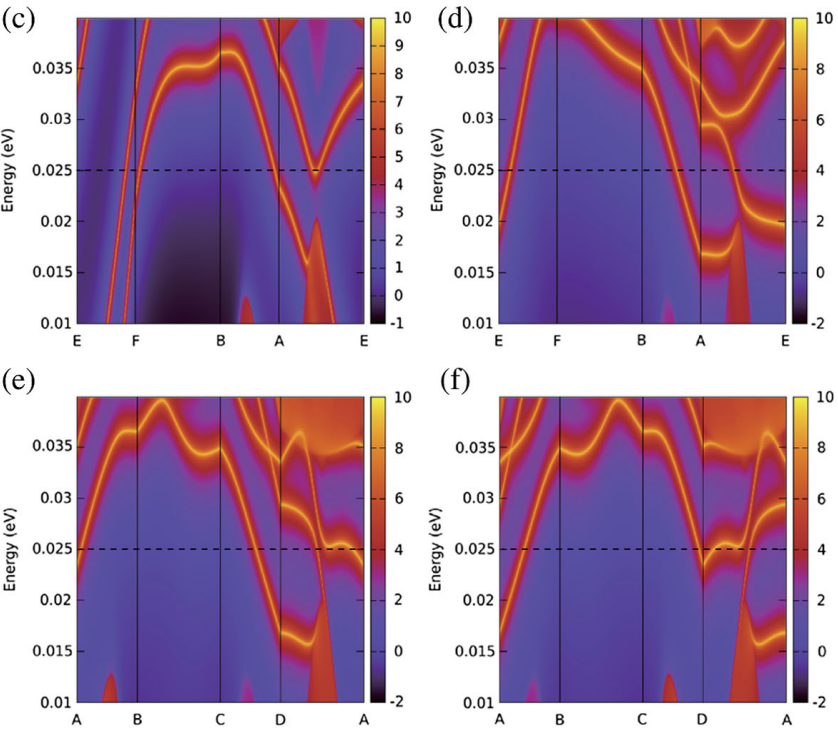

FIG. 8. Fermi surface of the second cleaved cell and the identification of the chirality of the surface state. (a,b) Fermi surface on top and bottom (010) surfaces, respectively. The small dots represent the single projected WP, and the large dots represent two WPs with the same chirality projecting on top of each other. Panels (c) and (e) are the surface states of the top surface along the closed paths of E-F-B-A-E and A-B-C-D-A, respectively. Panels (d) and (f) are the same as (c) and (e) but for the bottom surface. 
illustrated in Fig. 7. The termination of the first cleaved cell enclosed by the green dashed line is composed of cerium and tin atoms on one side and rhodium and tin atoms on the other side. The termination of the second cleaved cell enclosed by the red dashed line is composed of rhodium and tin atoms on both sides.

\section{APPENDIX B: SURFACE-STATE CALCULATION}

The Fermi arc patterns for both nonequivalent cleavage planes are calculated in the present study. The results for the first cleavage plane (illustrated by the green dashed line in Fig. 7) have already been plotted in Fig. 5 and discussed in the main text. Here, we further present the results for the second cleavage plane (illustrated by the

(a)

(010) top surface FS@0.025eV

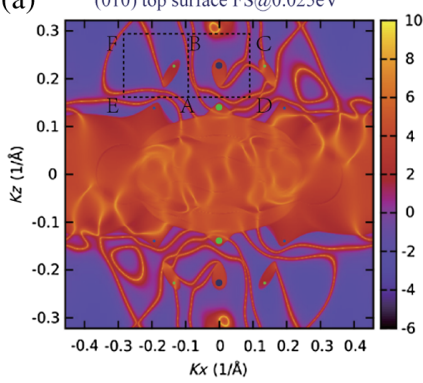

(c)
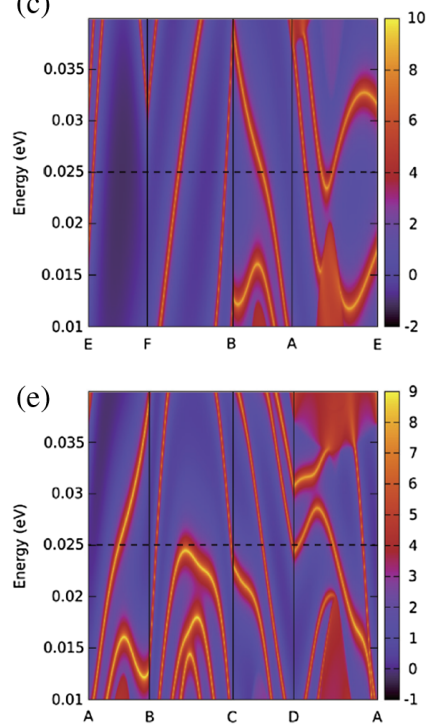

(b) (010) bottom surface FS@0.025eV

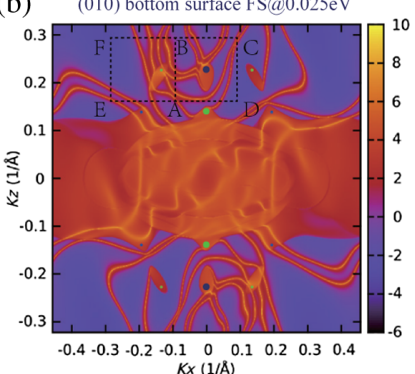

(d)
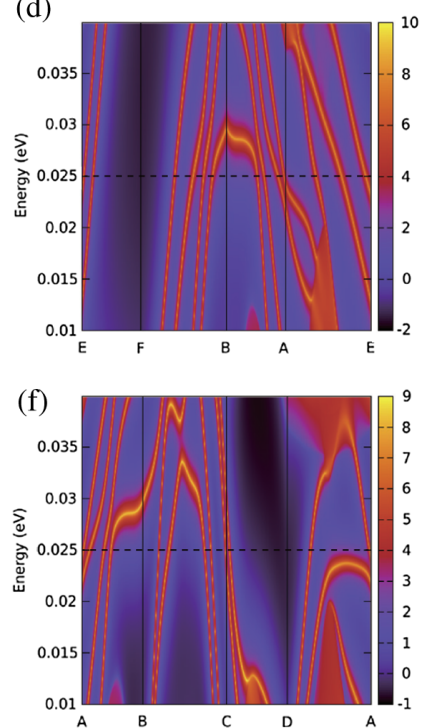

FIG. 9. Surface state of the first cleaved cell with a surface potential energy of $0.4 \mathrm{eV}$. (a,b) Fermi surface on the top and bottom (010) surfaces, respectively. The small dots represent the single projected WP, and the large dots represent two WPs with the same chirality projecting on top of each other. Panels (c) and (e) are the surface states of the top surface along the closed paths of E-F-B-A-E and A-B-C-D-A, respectively. Panels (d) and (f) are the same as (c) and (e) but for the bottom surface.

green dashed line in Fig. 7), which are plotted in Fig. 8. As introduced in detail in the main text, the summation of the chirality of all the Fermi crossing points along a closed loop in the surface BZ can be viewed as the surface topological invariance, which is fully determined by the bulk band structure but not the details on each particular surface. Here, we demonstrate this point by calculating the surface band structure on another cleavage plane. We can see from Fig. 8 that although the connection pattern of the Fermi arcs is changed completely, the abovedefined surface topological invariance remains the same. For instance, in Figs. 8(a) and 8(c), the loop E-F-B-A-E contains a single WP with chirality of +1 , so the summation of the chirality of the surface states along the loop is +1 . We can apply a similar analysis to loop A-B-C-D-A as well, where two WPs with total chirality -2 are projected inside the loop.
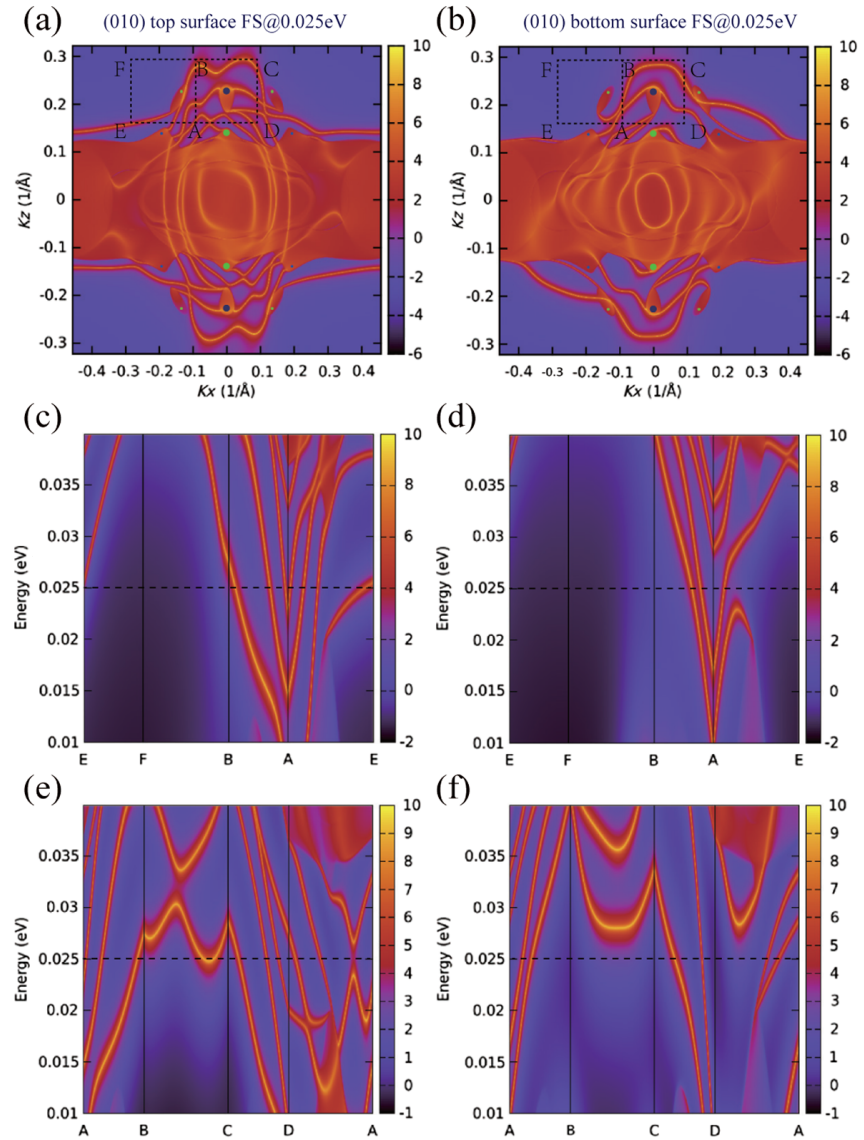

FIG. 10. Surface state of the first cleaved cell with surface potential energy of $-0.4 \mathrm{eV}$. (a,b) Fermi surface on the top and bottom (010) surfaces, respectively. The small dots represent the single projected WP, and the large dots represent two WPs with the same chirality projecting on top of each other. Panels (c) and (e) are the surface states of the top surface along the closed paths of E-F-B-A-E and A-B-C-D-A, respectively. Panels (d) and (f) are the same as (c) and (e) but for the bottom surface. 


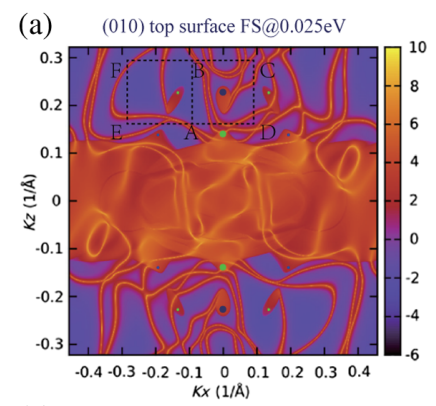

(c)

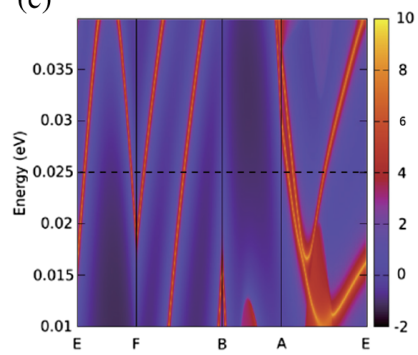

(e)

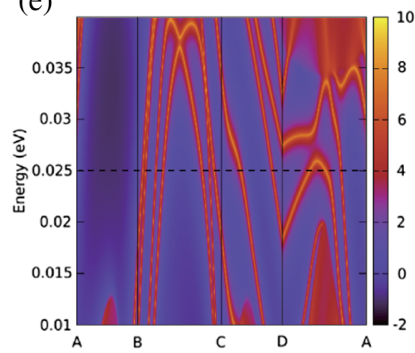

(b) (010) bottom surface FS@0.025eV

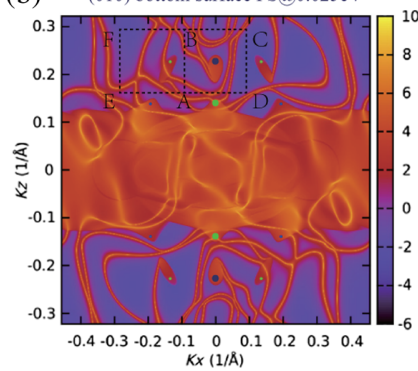

(d)

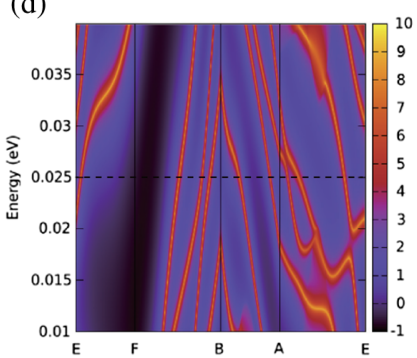

(f)

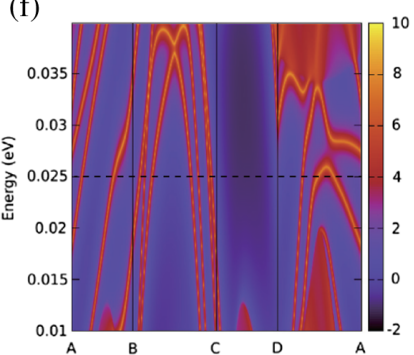

FIG. 11. Surface state of the second cleaved cell with a surface potential energy of $0.4 \mathrm{eV}$. (a,b) Fermi surface on the top and bottom (010) surfaces, respectively. The small dots represent the single projected WP, and the large dots represent two WPs with the same chirality projecting on top of each other. Panels (c) and (e) are the surface states of the top surface along the closed paths of E-F-B-A-E and A-B-C-D-A, respectively. Panels (d) and (f) are the same as (c) and (e) but for the bottom surface.

\section{APPENDIX C: EFFECTS OF SURFACE POTENTIAL}

The above calculations of the surface states are based on the tight-binding approximation without considering the possible surface potential caused by the charge redistribution near the surface area. To numerically study the effect of the surface potential, we include it in our tight-binding Hamiltonian as a parameter to see how the pattern of the Fermi arc evolves with the surface potential. In Figs. 9-11, and 12, we modify the surface potential from $-0.4 \mathrm{eV}$ to $0.4 \mathrm{eV}$ for the two different surface terminations described in the previous section. Our results clearly show that although the Fermi arc pattern changes dramatically by varying the surface potential, the topological invariance defined above remains unchanged, which demonstrates the robustness of the Fermi arcs on the (010) surface.
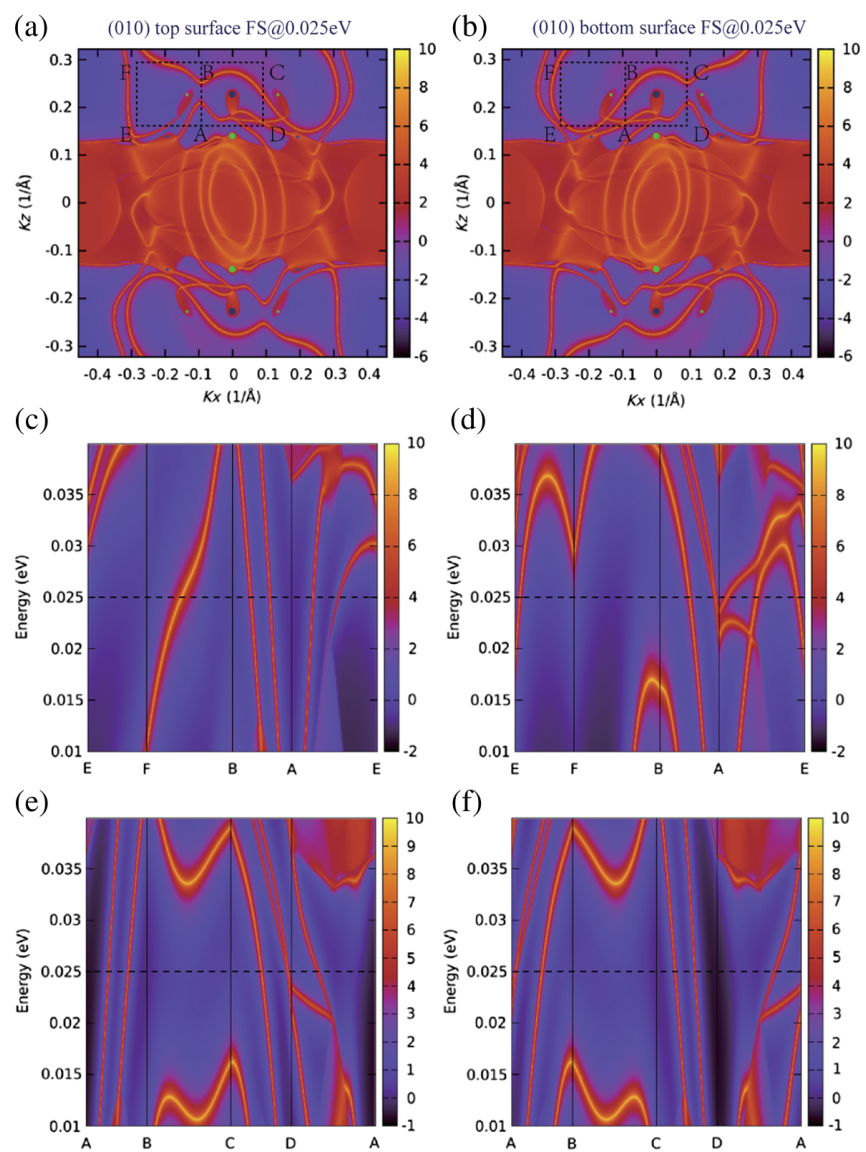

FIG. 12. Surface state of the second cleaved cell with a surface potential energy of $-0.4 \mathrm{eV}$. $(\mathrm{a}, \mathrm{b})$ Fermi surface on the top and bottom (010) surfaces, respectively. The small dots represent the single projected WP, and the large dots represent two WPs with the same chirality projecting on top of each other. Panels (c) and (e) are the surface states of the top surface along the closed paths of E-F-B-A-E and A-B-C-D-A, respectively. Panels (d) and (f) are the same as (c) and (e) but for the bottom surface.

[1] H. Weng, X. Dai, and Z. Fang, Topological Semimetals Predicted from First-Principles Calculations, J. Phys. Condens. Matter 28, 303001 (2016).

[2] X. Wan, A. M. Turner, A. Vishwanath, and S. Y. Savrasov, Topological Semimetal and Fermi-Arc Surface States in the Electronic Structure of Pyrochlore Iridates, Phys. Rev. B 83, 205101 (2011).

[3] G. Xu, H. Weng, Z. Wang, X. Dai, and Z. Fang, Chern Semimetal and the Quantized Anomalous Hall Effect in $\mathrm{HgCr}_{2} \mathrm{Se}_{4}$, Phys. Rev. Lett. 107, 186806 (2011).

[4] H. Weng, C. Fang, Z. Fang, B. A. Bernevig, and X. Dai, Weyl Semimetal Phase in Noncentrosymmetric TransitionMetal Monophosphides, Phys. Rev. X 5, 011029 (2015).

[5] S. M. Huang, S. Y. Xu, I. Belopolski, C. C. Lee, G. Chang, B. K. Wang, N. Alidoust, G. Bian, M. Neupane, C. Zhang, S. Jia, A. Bansil, H. Lin, and M. Z. Hasan, A Weyl Fermion Semimetal with Surface Fermi Arcs in the Transition 
Metal Monopnictide TaAs Class, Nat. Commun. 6, 7373 (2014).

[6] B. Q. Lv, H. M. Weng, B. B. Fu, X. P. Wang, H. Miao, J. Ma, P. Richard, X. C. Huang, L. X. Zhao, G. F. Chen, Z. Fang, X. Dai, T. Qian, and H. Ding, Experimental Discovery of Weyl Semimetal TaAs, Phys. Rev. X 5, 031013 (2015).

[7] Su-Yang Xu et al., Discovery of a Weyl Fermion Semimetal and Topological Fermi Arcs, Science 349, 613 (2015).

[8] B. Q. Lv et al., Observation of Weyl Nodes in TaAs, Nat. Phys. 11, 724 (2015).

[9] L. Yang, Z. Liu, Y. Sun, H. Peng, H. Yang, T. Zhang, B. Zhou, Y. Zhang, Y. Guo, M. Rahn et al., Weyl Semimetal Phase in the Non-Centrosymmetric Compound TaAs, Nat. Phys. 11, 728 (2015).

[10] Z. Wang, Y. Sun, X.-Q. Chen, C. Franchini, G. Xu, H. Weng, X. Dai, and Z. Fang, Dirac Semimetal and Topological Phase Transitions in $A_{3} \mathrm{Bi}(A=\mathrm{Na}, K, R b)$, Phys. Rev. B 85, 195320 (2012).

[11] Z. Wang, H. Weng, Q. Wu, X. Dai, and Z. Fang, ThreeDimensional Dirac Semimetal and Quantum Transport in $\mathrm{Cd}_{3} \mathrm{As}_{2}$, Phys. Rev. B 88, 125427 (2013).

[12] Z. K. Liu, B. Zhou, Y. Zhang, Z. J. Wang, H. Weng, D. Prabhakaran, S.-K. Mo, Z. X. Shen, Z. Fang, X. Dai, Z. Hussain, and Y. L. Chen, Discovery of a Three-Dimensional Topological Dirac Semimetal, $\mathrm{Na}_{3} \mathrm{Bi}$, Science 343, 864 (2014).

[13] Z. K. Liu, J. Jiang, B. Zhou, Z. J. Wang, Y. Zhang, H. M. Weng, D. Prabhakaran, S. K. Mo, H. Peng, P. Dudin, T. Kim, M. Hoesch, Z. Fang, X. Dai, Z.-X. Shen, D. L. Feng, Z. Hussain, and Y.L. Chen, A Stable Three-Dimensional Topological Dirac Semimetal $\mathrm{Cd}_{3} \mathrm{As}_{2}$, Nat. Mater. 13, 677 (2014).

[14] S. Borisenko, Q. Gibson, D. Evtushinsky, V. Zabolotnyy, B. Büchner, and R. J. Cava, Experimental Realization of a Three-Dimensional Dirac Semimetal, Phys. Rev. Lett. 113, 027603 (2014).

[15] S.-Y. Xu et al., Observation of Fermi Arc Surface States in a Topological Metal, Science 347, 294 (2015).

[16] R. Yu, H. Weng, Z. Fang, X. Dai, and X. Hu, Topological Node-Line Semimetal and Dirac Semimetal State in Antiperovskite $\mathrm{Cu}_{3} \mathrm{PdN}$, Phys. Rev. Lett. 115, 036807 (2015).

[17] Y. Kim, B. J. Wieder, C. L. Kane, and A. M. Rappe, Dirac Line Nodes in Inversion-Symmetric Crystals, Phys. Rev. Lett. 115, 036806 (2015).

[18] M. Dzero, K. Sun, V. Galitski, and P. Coleman, Topological Kondo Insulators, Phys. Rev. Lett. 104, 106408 (2010).

[19] M. Dzero, K. Sun, P. Coleman, and V. Galitski, Theory of Topological Kondo Insulators, Phys. Rev. B 85, 045130 (2012).

[20] F. Lu, J. Zhao, H. Weng, Z. Fang, and X. Dai, Correlated Topological Insulators with Mixed Valence, Phys. Rev. Lett. 110, 096401 (2013).

[21] D. J. Kim, S. Thomas, T. Grant, J. Botimer, Z. Fisk, and J. Xia, Robust Surface Hall Effect and Nonlocal Transport in $\mathrm{SmB}_{6}$ : Indication for an Ideal Topological Insulator, Sci. Rep. 3, 3150 (2013).

[22] J. Jiang, S. Li, T. Zhang, Z. Sun, F. Chen, Z. R. Ye, M. Xu, Q. Q. Ge, S. Y. Tan, X. H. Niu et al., Observation of In-Gap Surface States in the Kondo Insulator $\mathrm{SmB}_{6}$ by Photoemission, Nat. Commun. 4, 3010 (2013).
[23] N. Xu et al., Surface and Bulk Electronic Structure of the Strongly Correlated System $\mathrm{SmB}_{6}$ and Implications for a Topological Kondo Insulator, Phys. Rev. B 88, 121102(R) (2013).

[24] H. Weng, J. Zhao, Z. Wang, Z. Fang, and X. Dai, Topological Crystalline Kondo Insulator in Mixed Valence Ytterbium Borides, Phys. Rev. Lett. 112, 016403 (2014).

[25] M. Dzero, J. Xia, V. Galitski, and P. Coleman, Topological Kondo Insulators, Annu. Rev. Condens. Matter Phys. 7, 249 (2016).

[26] Z. Wang, X.-L. Qi, and S.-C. Zhang, Topological Order Parameters for Interacting Topological Insulators, Phys. Rev. Lett. 105, 256803 (2010).

[27] Z. Wang and S.-C. Zhang, Simplified Topological Invariants for Interacting Insulators, Phys. Rev. X 2, 031008 (2012).

[28] R. Pöttgen, R.-D. Hoffmann, E. V. Sampathkumaran, I. Das, B. D. Mosel, and R. Müllmann, Crystal Structure, Specific Heat, and ${ }^{119} \mathrm{Sn}$ Mössbauer Spectroscopy of $\mathrm{CeRu}_{4} \mathrm{Sn}_{6}$ : A Ternary Stannide with Condensed, Distorted $\mathrm{RuSn}_{6}$ Octahedra, J. Solid State Chem. 134, 326 (1997).

[29] I. Das and E. V. Sampathkumaran, Electrical-Resistance Anomalies in a Ce-Ru-Sn phase, Phys. Rev. B 46, 4250 (1992).

[30] E. M. Brüning, M. Brando, M. Baenitz, A. Bentien, A. M. Strydom, R. E. Walstedt, and F. Steglich, Low-Temperature Properties of $\mathrm{CeRu}_{4} \mathrm{Sn}_{6}$ from NMR and Specific Heat Measurements: Heavy Fermions Emerging from a Kondo-Insulating State, Phys. Rev. B 82, 125115 (2010).

[31] V. Guritanu et al., Anisotropic Optical Conductivity of the Putative Kondo Insulator $\mathrm{CeRu}_{4} \mathrm{Sn}_{6}$, Phys. Rev. B 87, 115129 (2013).

[32] M. Sundermann et al., $\mathrm{CeRu}_{4} \mathrm{Sn}_{6}:$ A Strongly Correlated Material with Nontrivial Topology, Sci. Rep. 5, 17937 (2015).

[33] P. Wissgott and K. Held, Electronic Structure of $\mathrm{CeRu}_{4} \mathrm{Sn}_{6}$ : A Density Functional Plus Dynamical Mean Field Theory Study, Eur. Phys. J. B 89, 5 (2016).

[34] R. Pöttgen, R.-D. Hoffmann, E. V. Sampathkumaran, I. Das, B. D. Mosel, and R. Müllmann, Crystal Structure, Specific Heat, and ${ }^{119} \mathrm{Sn}$ Mössbauer Spectroscopy of $\mathrm{CeRu}_{4} \mathrm{Sn}_{6}:$ A Ternary Stannide with Condensed, Distorted $\mathrm{RuSn}_{6}$ Octahedra, J. Solid State Chem. 134, 326 (1997).

[35] E. M. Brüning, M. Brando, M. Baenitz, A. Bentien, A. M. Strydom, R. E. Walstedt, and F. Steglich, Low-Temperature Properties of $\mathrm{CeRu}_{4} \mathrm{Sn}_{6}$ from NMR and Specific Heat Measurements: Heavy Fermions Emerging from a Kondo-Insulating State, Phys. Rev. B 82, 125115 (2010).

[36] X. Y. Deng, L. Wang, X. Dai, and Z. Fang, Local Density Approximation Combined with Gutzwiller Method for Correlated Electron Systems: Formalism and Applications, Phys. Rev. B 79, 075114 (2009).

[37] G. Kresse and J. Furthmüller, Efficient Iterative Schemes for Ab Initio Total-Energy Calculation Using a Plane-Wave Basis Set, Phys. Rev. B 54, 11169 (1996).

[38] A. A. Mostofi, J. R. Yates, G. Pizzi, Y.-S. Lee, I. Souza, D. Vanderbilt, and N. Marzari, An Updated Version of Wannier90: A Tool for Obtaining Maximally-Localised Wannier Functions, Comput. Phys. Commun. 185, 2309 (2014). 
[39] B. T. Thole, G. van der Laan, J. C. Fuggle, G. A. Sawatzky, R. C. Karnatak, and J.-M. Esteva, 3d X-Ray-Absorption Lines and the $3 d^{9} 4 f^{n+1}$ Multiplets of the Lanthanides, Phys. Rev. B 32, 5107 (1985).

[40] K. Held, Electronic Structure Calculations Using Dynamical Mean Field Theory, Adv. Phys. 56, 829 (2007).

[41] N. Lanatà, H. U. R. Strand, X. Dai, and B. Hellsing, Efficient Implementation of the Gutzwiller Variational Method, Phys. Rev. B 85, 035133 (2012).
[42] Y. X. Yao, C. Z. Wang, and K. M. Ho, Including Many-Body Screening into Self-Consistent Calculations: Tight-Binding Model Studies with the Gutzwiller Approximation, Phys. Rev. B 83, 245139 (2011).

[43] F. Y. Li, X. Luo, X. Dai, Y. Yu, F. Zhang, and G. Chen, Hybrid Weyl Semimetal, Phys. Rev. B 94, 121105 (2016).

[44] Q. S. Wu and S. N. Zhang, https://github.com/quanshengwu/ wannier_tools. 